\title{
Las bases neurales de la Amnesia Disociativa (AD): una revisión sistemática de la bibliografía
}

\author{
Systematic revision of the neural bases of dissociative amnesia
}

\author{
Carolina Cuesta', Florencia C. Cossini², Daniel G. Politis ${ }^{3}$
}

\begin{abstract}
Resumen
Introducción: La Amnesia Disociativa (AD) es una amnesia de tipo retrógrada caracterizada por una alteración en la memoria episódica. Objetivo: identificar el consenso científico actualizado sobre las bases neurales que subyacen al desarrollo de la amnesia disociativa. Metodología: revisión bibliográfica sistemática y evaluativa de tipo cualitativa. Resultados: La bibliografía encontrada sugiere inhibición funcional en hipocampo, amígdala, lóbulos temporales, corteza prefrontal y tálamo. Además, se observó hipoglucemia en la corteza cerebral derecha, en la unión fronto-temporal. Una inhibición del potencial de acción P300 también fue observada. Conclusión: Hay información suficiente para decir que la AD es una patología con base biológica objetivable. Se hace necesaria la revisión de la conceptualización actual de este síndrome amnésico y el planteamiento de nuevos criterios que distingan la AD de la orgánica.
\end{abstract}

Palabras clave: Amnesia disociativa - Bases neurales - Amnesia orgánica - Memoria episódica.

\begin{abstract}
Abtract
Introduction: Dissociative amnesia (DA) is a retrograde amnesia characterized by an alteration in episodic memory. Aim: Establish the neural bases which underlie the development of dissociative amnesia. Methods: Systematic and evaluative bibliographic review of qualitative type. Results: The bibliography found suggested functional inhibition in the hippocampus, amygdala, temporal lobes, prefrontal cortex and thalamus. Also, hypoglycemia was observed in right cerebral cortex, at the fronto-temporal junction. An inhibition in the potential action P300 was also stated. Conclusions: There is enough evidence to say that dissociative amnesia is an objectifiable biologically based pathology. There is a need to review the current conceptualization of this syndrome and to establish new criteria that would allow us to distinguish DA from organic amnesias.
\end{abstract}

Keywords: Dissociative amnesia - Neural bases - Organic amnesia - Episodic memory.

\footnotetext{
RECIBIDO II/2/202I - ACEPTADO 14/5/202।

'. Licenciada en Psicología. Becaria doctoral UBACyT. Laboratorio de deterioro cognitivo, Servicio de Neurología, Hospital Interzonal de Agudos “Eva Perón" (CONICET).

2. Doctora en Psicología. Becaria posdoctoral CONICET. Laboratorio de deterioro cognitivo (CONICET), Servicio de Neurología, Hospital Interzonal General de Agudos “Eva Perón”.

3. Médico neurólogo. Doctor en Medicina. Investigador independiente ad honorem CONICET; Laboratorio de deterioro cognitivo, Servicio de neurología, Hospital Interzonal General de Agudos "Eva Perón".
}

Autora de referencia:

Carolina Cuesta caro.cuesta.cc@gmail.com

Lugar de realización del estudio: Laboratorio de deterioro cognitivo, servicio de neurología, Hospital Interzonal de Agudos “Eva Perón” (CONICET), Prov. de Buenos Aires, Argentina. 


\section{Introducción}

La amnesia disociativa $(\mathrm{AD})$, también denominada psicógena o funcional, es un síndrome clasificado dentro de los trastornos disociativos, los cuales se caracterizan por una disrupción y/o discontinuidad en la integración normal de conciencia, memoria, identidad, emoción, percepción, representación corporal, control motor y conducta. La $\mathrm{AD}$ es la incapacidad para recuperar información autobiográfica, usualmente provocado por un evento de naturaleza traumática o estresante. Es inconsistente con el olvido normal y no puede ser mejor explicada por efectos fisiológicos debido a sustancias, enfermedades neurológicas $\mathrm{u}$ otras, trastorno disociativo de identidad, trastorno por estrés post-traumático, trastorno de estrés agudo, trastorno de síntomas somáticos y/o trastorno neurocognitivo leve o mayor (American Psychiatric Association, 2013; Loewenstein, 2018). La amnesia impide la expresión emocional y bloquea la reevaluación cognitiva, llevando así a conductas de evitación (Echeburúa \& Corral, 2007). Los síntomas causan malestar clínicamente significativo y llevan a un deterioro en lo social, en lo ocupacional y en otras áreas importantes del funcionamiento del sujeto. Su cuadro es de comienzo súbito y finalización abrupta, con recuperación ad integrum y escasas recurrencias e incluye amnesia de identidad y humor deprimido (American Psychiatric Association, 2013).

Teorías que intentaron sentar las bases de esta patología pusieron el foco en factores ambientales-psicológicos, excluyéndose así a factores biológicos (Staniloiu et al., 2018). De allí surge la diferenciación entre amnesias disociativas y amnesias orgánicas (AO), utilizándose como criterio de separación la presencia o ausencia de bases biológicas. La crítica a este criterio ha llevado a su transformación, estableciéndose como nueva línea de separación la presencia o ausencia de bases biológicas objetivas y medibles (Ventura, 1993; López et al., 2017).

Ambos tipos de amnesia presentan perfiles cognitivos diferentes. La AD presenta compromiso severo de la memoria episódica con amnesia retrógrada y, en menor medida, anterógrada; pérdida de la identidad; alteración en memoria semántica, en lenguaje (presencia de anomias), en habilidades procedurales $\mathrm{y}$, con menor frecuencia, compromiso de las habilidades intelectuales. En la AO se encuentra severamente comprometida la memoria episódica con amnesia anterógrada $\mathrm{y}$, en menor medida, retrógrada, habiendo conservación de los sistemas de memoria restantes, de la identidad y de las habilidades intelectuales (Serra et al., 2007). En la actualidad, son muy vagos los criterios con los cuales se cuenta para el diagnóstico diferencial entre AD y AO. No es sino hasta evolucionado el cuadro patológico que se puede diferenciar entre ambas amnesias.

El presente trabajo tiene como objetivo establecer las bases neurales de la $\mathrm{AD}$, exponiendo las principales áreas cerebrales que se ven afectadas en esta patología y que llevan al desarrollo de la misma.

\section{Metodología}

Se realizó una revisión sistemática y evaluativa de tipo cualitativa y de carácter explicativo-analítico. Dos revisores llevaron a cabo el proceso de forma independiente. Se incluyeron publicaciones científicas que hayan expuesto en sus resultados las bases neurales de la $\mathrm{AD}$. Se excluyeron aquellos estudios que han utilizado métodos experimentales, tesis de grado y publicaciones no oficiales. Todos los trabajos incluidos en esta revisión fueron publicados en revistas indexadas. Se consultaron las bases de datos Science Direct, Pubmed, Psychinfo y Scielo y se utilizaron las siguientes palabras claves: "amnesia disociativa" (psychogenic amnesia), con sus derivados "amnesia psicógena" (dissociative amnesia) y "amnesia funcional" (functional amnesia); "amnesia retrógrada" (retrograde amnesia); "memoria episódica" (episodicmemory); "bases neurales" (neural bases); "neuroimágenes" (neuroimaging); y "trauma psíquico" (psychic trauma).

$\mathrm{Al}$ inicio del proceso de revisión, se le entregó a cada revisor una planilla con información acerca del objetivo de la búsqueda, criterios de inclusión y de exclusión, palabras claves a utilizar y bases de datos a consultar. Luego, cada uno comenzó la revisión, basando su selección en los títulos y resúmenes de los artículos encontrados. Se encontraron un total de 41 artículos. Ambos revisores, en conjunto, analizaron los estudios seleccionados y aquellos que generaron dudas respecto a su inclusión o exclusión de esta revisión fueron leídos de forma completa. De los 41 hallados, 7 fueron excluidos por no cumplir los criterios para este estudio. En última instancia, los artículos restantes fueron leídos en su totalidad. En esta etapa no se excluyó ningún trabajo. Esta revisión incluye 34 estudios.

\section{Bases neurales de la AD}

En la $\mathrm{AD}$ se observa una alteración orgánica en las principales estructuras cerebrales que participan tanto del procesamiento de la memoria como del procesamiento de información con contenido emocional. Estas estructuras son: corteza frontal, lóbulos tempo- 
rales (hipocampo, amígdala, lóbulo temporal medial, lóbulo temporal derecho y lóbulo temporal izquierdo) y diencéfalo (tálamo e hipotálamo) (Brandt \& Van Gorp, 2006; Farina et al. 2014, Kunii et al. 2012; Staniloiu \& Markowitsch, 2010; Staniloiu et al., 2012; Van der Kolk, 2015).

\section{Liberación de neuropéptidos y neuro- transmisores}

Los mecanismos que organizan la respuesta del cuerpo frente a una situación estresante conllevan no sólo la activación de áreas específicas del cerebro, como hipocampo, amígdala y corteza prefrontal, sino también la liberación de determinados neurotransmisores y neuropéptidos moduladores del estrés (Dirven et al., 2017). Un nivel de estrés mínimo ya es suficiente para provocar una alteración en los niveles de concentración de la dopamina, serotonina, acetilcolina y noradrenalina, principalmente, en las áreas de la corteza prefrontal, amígdala e hipocampo. (Asan et al., 2013; Lataster et al., 2011; Mei et al., 2020).

En situaciones de estrés agudo, se producen cambios en los niveles de la corticosterona, capaz de modificar la actividad de los neurotransmisores en las áreas mencionadas (Mora et al., 2012). Los dos receptores de la corticosterona son el mineralocorticoides y el glucocorticoides, ambos distribuidos a través de la amígdala, el hipocampo y la corteza prefrontal, principalmente. Es la acción de la corticosterona por sobre los neurotransmisores lo que lleva, entre otras cosas, a la consolidación de la memoria a largo plazo de eventos de esta naturaleza.

La liberación alterada de estos neuropéptidos y neurotransmisores está asociada a la incapacidad de recordar el evento estresante y a adoptar conductas disruptivas que llevan al malestar clínico del sujeto y, eventualmente, al desarrollo de la AD (De Renzi, 2002; Kumar et al., 2017; Markowitsch et al., 1997).

\section{Hipermetabolismo e hipometabolismo Estudios con fMRI}

Hay investigaciones realizadas con fMRI (fMRI, por su siglas en inglés Functional Magnetic Resonance Imaging o Resonancia Magnética Funcional) que sostienen que los amnésicos disociativos, durante el procesamiento de información, presentan en simultáneo una activación y una inhibición en el funcionamiento de ciertas regiones cerebrales que difieren de aquellas observadas en sujetos sanos. Se ha encontrado un hipermetabolismo en el precúneo izquierdo (Markowitsch et al., 1997); región parietal posterior izquierda y derecha (Markowitsch et al., 1997; Arzy et al., 2011); área prefrontal dorsal derecha y área prefrontal lateral inferior y medial izquierda (Kunii et al., 2012; Markowitsch et al., 1997; Yasuno et al., 2000; Kikuchi et al., 2010); corteza cingulada derecha (Markowitsch et al., 1997; Yasuno et al., 2000); lóbulo temporal medial anterior derecho (incluida la amígdala), corteza occipital izquierda, putamen derecho y cerebelo derecho (Yasuno et al., 2000); hipocampo izquierdo (Kapur et al., 1995); y corteza frontotemporal izquierda (Fink et al., 1996; Markowitsch, 1996). Otros estudios entran en contradicción con éstos, hallando un hipometabolismo en algunas de las áreas recién expuestas: putamen derecho, cerebelo derecho (Markowitsch et al., 1997) e hipocampo izquierdo (Kikuchi et al., 2010; Arzy et al., 2011). También se encontró un hipometabolismo en el girus temporal medio e inferior superior izquierdo (Markowitsch et al., 1997; Kikuchi et al., 2010); tálamo derecho (Markowitsch et al., 1997); lóbulo parietal inferior bilateral (Kunii et al., 2012); lóbulo temporal superior izquierdo e inferior (Kunii et al., 2012; Kikuchi et al., 2010) y la corteza insular (Brand et al., 2009). Múltiples regiones cerebrales involucradas en el procesamiento de la memoria y emoción se ven inhibidas en su función, produciendo esto un tratamiento deficitario de la información y trayendo como consecuencia que otras áreas se activen en su reemplazo.

\section{Estudios con PET}

Al estudiar el nivel de concentración de glucosa en sangre a partir de estudios con PET (PET por su nombre en inglés, Positron Emission Tomography, o Tomografía por Emisión de Positrones), se encontró hipometabolismo en el hemisferio derecho de la corteza cerebral, en la unión frontotemporal derecha y en la corteza prefrontal inferolateral derecha (Brandt \& Van Gorp, 2006; Markowitsch et al., 1997; Markowitsch, 1999).

\section{Estudios con SPECT}

En estudios con SPECT (SPECT por su nombre en inglés, Single Photon Emission Computed Tomography o Tomografía Computarizada por Emisión de Fotón Único) que evaluaron los niveles de perfusión, se obtuvieron resultados que muestran un nivel bajo en las regiones frontal y temporal derechas al procesar información de contenido emocional (Markowitsch et al., 1997). Se hallaron patrones de cambios secuenciales específicos en el flujo sanguíneo cerebral durante el proceso de recuperación de información: a) incremento transitorio en el flujo sanguíneo durante la etapa de recuperación de información, seguido por 
otro incremento luego de finalizado el proceso, b) incremento transitorio en el flujo sanguíneo durante la etapa de recuperación de información, manteniéndose su nivel constante o decreciendo en las regiones parietal, angular, central, pericallosal e hipocampal luego de finalizado el proceso y c) niveles constantes de flujo sanguíneo durante todo el proceso o decreciendo en el lóbulo temporal, el ganglio basal, el tálamo y el cerebelo luego de finalizada la etapa de recuperación de información (Arzy et al., 2011).

\section{Hipótesis de desincronización}

En diversos estudios se ha encontrado que el desarrollo de la $\mathrm{AD}$ de debe a una desincronización en los procesos de acceso a los patrones de memoria (Staniloiu et al., 2012; Markowitsch, 1996; Sar, 2006). En sujetos sanos, se da una sincronización de la actividad neuronal para recuperar la información almacenada; se activan y se sincronizan áreas cerebrales específicas, como la corteza temporal anterior derecha, la corteza prefrontal y regiones del circuito límbico basolateral (la región anterior ventral, la amígdala y el tálamo medial).

En los amnésicos disociativos ocurre una desincronización que lleva a la interrupción, desconexión o bloqueo en la red neuronal implicada en la recuperación de la memoria episódica, con la consecuente inhabilitación a alcanzar la información previamente almacenada. La $\mathrm{AD}$, por lo tanto, estaría causada por un déficit en la recuperación de información, mientras se mantiene conservado el almacenamiento de la misma.

\section{Memoria explícita almacenada como memoria implícita}

Ciertos autores postulan que los amnésicos disociativos almacenan la información episódica como memoria implícita en vez de procesarla como memoria explícita (Van der Kolk, 2015; Campodonico \& Rediess, 1996; Rothschild, 2000). Se observó, a partir de tareas indirectas de memoria, que los pacientes almacenaban, e incluso utilizaban, la información que se les requería, pero fallaban en evocarla de forma explícita. Anderson, Ochsner y Kuhl (2004) establecieron que, si se hace un uso repetido de este mecanismo, se produce una inhibición en el proceso de recuperación de la memoria.

\section{Estudios con potenciales relacionados con eventos}

De los trabajos que midieron potenciales de acción, los más estudiados fueron el P300 y el N100 (Farina et al., 2014; Fukuzako et al., 1999; Kirino, 2006).
El P300 está involucrado en múltiples funciones cognitivas, incluida la memoria (Amin et al., 2015; Oliveira et al., 2011). El P300 está distribuido en el hipocampo, en el giro parahipocampal, en el tálamo, en la corteza frontal inferior y en el lóbulo parietal (Linden, 2005; Ludowig et al., 2010). Por su parte, la corteza orbitofrontal, involucrada en la recuperación de información y en el procesamiento de estímulos emocionales, produce una amplitud en el P300 (Linden, 2005; Rule et al., 2002).

Se ha observado en pacientes una menor amplitud durante la etapa amnésica, en comparación a la observada en sujetos sanos. No se hallaron diferencias significativas entre pacientes y sujetos sanos en el N100.

El descenso de amplitud en el P300 provocaría un déficit en el sistema de memoria de estos sujetos, dificultando la integración de la información, la evocación de recuerdos pasados (Amin et al., 2015; Oliveira et al., 2011) y la producción de respuestas frente a estímulos con carga emocional (Rule et al., 2002).

\section{Conclusiones}

Esta revisión bibliográfica pone en evidencia las alteraciones neurales subyacentes a la $\mathrm{AD}$. Las alteraciones halladas en este estudio son de tipo funcional, no hallándose cambios a nivel estructural, e incluyen: a) alteraciones en los procesos mnésico y emocional; la disfunción de los amnésicos disociativos se concentra en los patrones de procesamiento de los sistemas de memoria y emoción, los cuales difieren de aquellos estudiados en sujetos sanos; b) alteraciones funcionales en distintas estructuras del SNC; se han hallado anormalidades funcionales, principalmente, en lóbulos temporales, hipocampo, amígdala, corteza prefrontal, corteza cerebral derecha y en la corteza frontotemporal.

Encontramos en los artículos revisados una coincidencia entre las estructuras cerebrales alteradas en los pacientes evaluados y la sintomatología clínica expuesta por el DSM V. Tanto la corteza prefrontal como los lóbulos temporales (aunque éstos en menor medida) participan de la recuperación de información. Las disfunciones neurales observadas darían sustento a la dificultad de los amnésicos psicógenos para recuperar la información episódica almacenada, no encontrándose afectada su capacidad para consolidar la misma.

Si bien los límites de las amnesias orgánicas-amnesias disociativas se tornan cada vez más borrosos, esta clasificación sigue estando presente en textos bibliográficos y en manuales de clasificación de patologías (American Psychiatric Association, 2013). 
En la actualidad, no contamos con indicadores que nos permitan establecer desde las primeras etapas de la enfermedad la diferenciación entre ambas amnesias. Es únicamente al avanzar el cuadro a estadios más tardíos que se va a poder realizar el diagnóstico diferencial. Estos indicadores están basados exclusivamente en el perfil cognitivo del paciente, no contando con ningún marcador biológico que permita, con mayor precisión, establecer el diagnóstico. Se hace necesaria la construcción de nuevos criterios, más eficaces y con mayor base científica, que permitan hacer el diagnóstico diferencial entre las $\mathrm{AD}$ y las $\mathrm{AO}$.

A partir de los estudios hallados en esta revisión, creemos que se podrían llegar a establecer como criterios de diagnóstico diferencial los perfiles cognitivos característicos a ambos tipos de amnesia y la presencia de determinados marcadores biológicos (fisiopatología de la AP), observados en distintos estudios funcionales (fMRI, PET, SPECT, PRE, entre otros).

Consideramos relevante seguir estudiando las bases biológicas de la $\mathrm{AD}$ para el establecimiento de criterios más uniformes y profundizar sobre el perfil neurocognitivo predominante, intentando dar respuesta a la pregunta de si este síndrome corresponde a un déficit de almacenamiento o de recuperación de la información.

\section{Conflictos de intereses: los autores declaran no tener} conflictos de intereses.

Agradecimientos: Agradecemos a la Facultad de Psicología de la Universidad de Buenos Aires y al Consejo Nacional de Investigaciones Científicas y Técnicas (CONICET) de Argentina.

\section{Referencias bibliográficas}

American Psychiatric Association. (2013). Diagnostic and Statistical Manual of Mental Disorders ( $5^{\circ}$ ed). American Psychiatryc Publishing.

Amin, H. U., Malik, A. S., Kamel, N., Chooi, W. T., Hussain, M. (2015). P300 correlates with learning \& memory abilities and fluid intelligence. J Neuroeng Rehabil, 12(1), 87. https://doi.org/10.1186/s12984-015-0077-6 Anderson, M. C., Ochsner, K. N., Kuhl, B., Cooper, J., Robertson, E., Gabrieli, S. W., Glover, G. H., Gabrieli, J. D. E. (2004). Neural systems underlying the suppression of unwanted memories. Sci, 303(5655), 232-235. doi: $10.1126 /$ science. 1089504

Arzy, S., Collette, S., Wissmeyer, M., Lazeyras, F., Kaplan, P. W., Blanke, O. (2011). Psychogenic amnesia and self-identity: a multimodal functional investigation. Eu J Neurol, 18(12), 1422-1425. https://doi.org/10.1111/ j.1468-1331.2011.03423.x

Asan, E., Steinke, M., Lesch, K. P. (2013). Serotonergic innervation of the amygdala: targets, receptors, and implications for stress and anxiety. Histochem Cell Biol; 139(6): 785-813. doi: 10.1007/s00418-013-1081-1

Brandt, J., Van Gorp, W.G. (2006). Functional (psychogenic) amnesia. En D. M. Greer, S. Pollandt, T. Bleck (Edits). Seminars in Neurolog. (pp. 331340). Thieme Medical Publishers.
Brand, M., Eggers, C., Reinhold, N., Fujiwara, E., Kessler, J., Heiss, W. D., Markowitsch, H. J. (2009). Functional brain imaging in 14 patients with dissociative amnesia reveals right inferolateral prefrontal hypometabolism. Psychiatry Res Neuroimaging, 174(1), 32-39. doi: 10.1016/j.pscychres$\underline{\text { ns.2009.03.008 }}$

Campodonico, J. R., Rediess, S. (1996). Dissociation of implicit and explicit knowledge in a case of psychogenic retrograde amnesia. J Clin Exp Neuropsychol, 2(2), 146-158. doi: 10.1017/s1355617700001004

De Renzi, E. (2002). What does psychogen mean? Cortex, 38(4), 678-681. doi: 10.1016/s0010-9452(08)70036-4

Dirven, B. C. J., Homberg, J. R., Kozicz, T., Henckens, M. J. A. G. (2017). Epigenetic programming of the neuroendocrine stress response by adult life stress. J Mol Endocrinol, 59(1), R11-R31. doi: 10.1530/JME-17-0019

Echeburúa, E., Corral, P.D. (2007). Intervención en crisis en víctimas de sucesos traumáticos: ¿Cuándo, cómo y para qué? Psicol Conductual, 15(3), 373-387.

Farina, B., Speranza, A. M., Dittoni, S., Gnoni, V., Trentini, C., Vergano, C. M. Liotti, G., Brunetti, R., Testani, E., Giacomo Della Marca, G. (2014). Memories of attachment hamper EEG cortical connectivity in dissociative patients. Eur Arch Psy Clin; 264(5), 449-458. doi: 10.1007/s00406-013$\underline{0461-9}$

Fink, G. R., Markowitsch, H. J., Reinkemeier, M., Bruckbauer, T., Kessler, J., Heiss, W. D. (1996). Cerebral representation of one's own past: neural networks involved in autobiographical memory. J Neurosci, 16(13), 42754282. doi: 10.1523/JNEUROSCI.16-13-04275.1996

Fukuzako, H., Fukuzaki, S., Fukuzako, T., Jing, H., Ueyama, K., Takigawa, M. (1999). P300 event-related potentials in probable dissociative generalized amnesia. Prog Neuro-psychopharmacol Biol Psychiatry, 23(8),1319-27. doi: 10.1016/s0278-5846(99)00068-8

Kapur, N., Friston, K. J., Young, A., Frith, C. D., Frackowiak, R. S. J. (1995). Activation of human hippocampal formation during memory for faces: a PET study. Cortex, 31(1), 99-108. doi: 10.1016/s0010-9452(13)80108-6

Kikuchi, H., Fujii, T., Abe, N., Suzuki, M., Takagi, M., Mugikura, S., Takahashi, S., Mori, E. (2010). Memory repression: brain mechanisms underlying dissociative amnesia. J Cogn Neurosci, 22(3), 602-613. doi: 10.1162/ jocn.2009.21212

Kirino, E. (2006). P300 is attenuated during dissociative episodes. J Nerv Ment Dis, 194(2), 83-90. doi: 10.1097/01.nmd.0000198145.18457.28

Kumar, S., Rao, S. L., Sunny, B., Gangadhar, B.N. (2017). Widespread cognitive impairment in psychogenic anterograde amnesia. Psychiatry Clin Neurosci, 61(6), 583-586. doi: 10.1111/j.1440-1819.2007.01735.x

Kunii, Y., Okano, T., Mashiko, H., Yabe, H., Niwa, S. I. (2012). Serial changes in cerebral blood flow single photon emission computed tomography findings during memory retrieval in a case of psychogenic amnesia. Psychiatry Clin Neurosci, 66(7), 623-624. doi: 10.1111/j.1440-1819.2012.02408.x

Lataster, J., Collip, D., Ceccarini, J., Haas, D., Booij, L., van Os, J., Pruessner, J., Van Laere, K., Myin-Germeys, I. (2011). Psychosocial stress is associated with in vivo dopamine release in human ventromedial prefrontal cortex: a positron emission tomography study using fallypride. Neuroimage, 58(4), 1081-1089. doi: 10.1016/j.neuroimage.2011.07.030

Linden, D.E. (2005). The P300: where in the brain is it produced and what does it tell us? Neuroscientist, 11(6), 563-576. doi: $\underline{10.1177 / 1073858405280524}$

Loewenstein, R. J. (2018). Dissociative amnesia: Epidemiology, pathogenesis, clinical manifestations, course, and diagnosis. Hentet; 10:20.

López, R.G., Garcés, J.G., Jiménez, J. G., Negro, J. M., Gutiérrez-Rojas, L. (2017). A case for considering differences between organic and psychogenic amnesia. Eur Psychiatry, 41(S1): Abstract of the 25th European Congress of Psychiatry, pp. S683 DOI: https://doi.org/10.1016/j.eurpsy.2017.01.1187

Ludowig, E., Bien, C. G., Elger, C. E., Rosburg, T. (2010). Two P300 generators in the hippocampal formation. Hippocampus, 20(1), 186-195. doi: $\underline{10.1002 / \text { hipo. } 20603}$ 
Markowitsch, H. J., Calabrese, P., Fink, G. R., Durwen, H. F., Kessler, J., Härting, C., König, M., Mirzaian, E. B., Heiss, W. D., Heuser, L., Gehlenb, W. (1997). Impaired episodic memory retrieval in a case of probable psychogenic amnesia. Psychiatry Res Neuroimaging, 74(2), 119-126. https:// doi.org/10.1016/S0925-4927(97)03041-2

Mei, L., Zhou, Y., Sun, Y., Liu, H., Zhang, D., Liu, P., Shu, S. (2020). Acetylcholine Muscarinic Receptors in Ventral Hippocampus Modulate Stress-Induced Anxiety-Like Behaviors in Mice. Front Mol Neurosci, 13:598811. https://doi.org/10.3389/fnmol.2020.598811

Mora, F., Segovia, G., del Arco, A., de Blas, M., Garrido, P. (2012). Stress, neurotransmitters, corticosterone and body-brain integration. Brain research, 1476: 71-85. doi: 10.1016/j.brainres.2011.12.049

Markowitsch, H. J. (1996). Organic and psychogenic retrograde amnesia: two sides of the same coin? Neurocase, 2(4), 357-371. https://doi. org $/ 10.1080 / 13554799608402410$

Markowitsch, H. J., Fink, G. R., Thone, A., Kessler, J., Heiss, W. D. (1997). A PET study of persistent psychogenic amnesia covering the whole life span. Cognitive Neuropsychiatry, 2(2), 135-158. doi: 10.1080/135468097396379

Markowitsch, H. J. (1999). Functional neuroimaging correlates of functional amnesia. Memory, 7(5-6), 561-584. doi: 10.1080/096582199387751

Oliveira, A. M., Estévez, M. A., Hawk, J. D., Grimes, S., Brindle, P. K., Abel, T. (2011). Subregion-specific p300 conditional knock-out mice exhibit long-term memory impairments. Learn Mem, 18(3), 161-169. doi: 10.1101/lm.1939811

Rothschild, B. (2000). The body remembers continuing education test: The psychophysiology of trauma \& trauma treatment. Norton \& Company.

Rule, R. R., Shimamura, A. P., Knight, R.T. (2002). Orbitofrontal cortex and dynamic filtering of emotional stimuli. Cogn Affect Behav Neurosci, 2(3), 264-270. doi: 10.3758/cabn.2.3.264
Sar, V. (2006). The scope of dissociative disorders: an international perspective. Psychiatr Clin, 29(1), 227-244. doi: 10.1016/j.psc.2005.1

Serra, L., Fadda, L., Buccione, I., Caltagirone, C., Carlesimo, G.A. (2007). Psychogenic and organic amnesia. A multidimensional assessment of clinical, neuroradiological, neuropsychological and psychopathological features. Behav Neurol; 18(1): 53-64. doi: 10.1155/2007/193140.

Staniloiu, A., Markowitsch, H. J. (2010). Searching for the anatomy of dissociative amnesia. J Psychol; 218(2), 96-108. doi: 10.1027/0044-3409/ a000017, 1 .

Staniloiu, A., Vitcu, I., Markowitsch, H. J. (2012). Neuroimaging and dissociative disorders. En J. M. Morihisa (Ed.) Advances in brain imaging. (pp. 11-34). InTech.

Staniloiu A, Markowitsch HJ, Kordon A. (2018). Psychological causes of autobiographical amnesia: A study of 28 cases. Neuropsychologia; 110: 134147. doi: 10.1016/j.neuropsychologia.2017.10.017

Van der Kolk B. A. (2015). The body keeps the score: Brain, mind, and body in the healing of trauma. Penguin Books.

Ventura, R. (1993). Amnesias psicógenas. En J. F. Dalmás (Ed.). La memoria desde la neuropsicología. (pp.210-223). Roca viva.

Yasuno, F., Nishikawa, T., Nakagawa, Y., Ikejiri, Y., Tokunaga, H., Mizuta, I., Shinozaki, K., Hashikawa, K., Sugita, Y., Nishimura, T., Takeda, M. (2000). Functional anatomical study of psychogenic amnesia. Psychiatry Res Neuroimaging, 99(1), 43-57. doi: 10.1016/s0925-4927(00)00057-3 